\title{
El desafío del cambio de la vacuna inactivada contra poliomielitis en América Latina. Declaración de la Sociedad Latinoamericana de Infectología Pediátrica (SLIPE)
}

\author{
Luiza Helena Falleiros-Arlant, María Luisa Avila-Agüero, José Brea del Castillo y Cristina Mariño
}

The challenge of changing the inactivated poliomyelitis vaccine in Latin America. Declaration of the Latin American Society of Pediatric Infectious Diseases (SLIPE)

Even though we have already covered $99 \%$ of the path to eradicate poliomyelitis from the world, this disease is still causing paralysis in children. Its eradication means not only the end of wild poliovirus circulation, but vaccine-derived poliovirus circulation as well. Taking into account different factors such as: current epidemiological data, adverse events of the attenuated oral poliomyelitis vaccine (OPV), the availability of an injectable inactivated vaccine (IPV) without the potential of causing the severe adverse events of the oral vaccine (OPV), the efficacy and effectiveness of the IPV in several countries of the world where it has been used for several years, the rationale of changing the vaccination schedule in different Latin American countries; the Latin American Society of Pediatric Infectious Diseases (SLIPE) announces its recommendation of switching to IPV in Latin America, by this Declaration, with an Action Plan for 2014-2015 period as regards vaccination against polio policies in Latin America. 1. The optimal proposed schedule consists of four IPV doses (three doses in the primary schedule plus a booster dose), whether IPV is combined or not with other indicated vaccines in the immunization program of the country. During the OPV to IPV transition phase, an alternative schedule is acceptable; 2 . Countries should set optimal strategies in order to maintain and improve vaccination coverage, and implement a nominal immunization registry; 3. Improving the Epidemiological Surveillance of Acute Flaccid Paralysis (AFP) and setting up an environmental surveillance program; 4. Setting up strategies for introducing IPV in National Immunization Programs, such as communicating properly with the population, among others; 5 . Bringing scientific societies closer to decision makers; 6. Ensuring optimal supply and prices for IPV introduction; 7. Training vaccination teams; 8. Enhancing the distribution and storing logistics of vaccines. In addition to the scientific evidence, the countries that have not yet decided to switch to IPV should consider the implications of equity and social justice.

Key words: Poliomyelitis, Polio vaccines, Polio vaccination schedules, Attenuated polio vaccine (OPV), Inactivated polio vaccine (IPV), Polio in Latin America.

Palabras clave: Poliomielitis, Vacunas contra la polio, Esquema de vacunación contra la polio, Vacuna atenuada anti-polio (OPV), Vacuna inativada anti-polio (IPV), Polio en América Latina, Erradicación de la polio, Vacuna oral contra la polio.

\section{Introducción}

L a poliomielitis (polio) continúa causando parálisis en niños. A diferencia de la mayoría de las enfermedades, la polio puede ser erradicada por completo. Ya hemos recorrido el $99 \%$ del camino para erradicarla del mundo. La erradicación de esta enfermedad significa el fin de la circulación de todos los virus de polio, tanto del salvaje como del virus derivado de la vacunación.

En 2014, solamente quedan tres países en los que no se ha podido detener la transmisión de la polio. Debemos tomar conciencia de esta oportunidad histórica para ganar esta batalla. La Organización Mundial de la Salud (OMS) cuenta con vacunas de bajo costo y eficaces para prevenir la polio. Debemos mantener el foco y la determinación, ya que disponemos de las herramientas y el conocimiento técnico para erradicar el virus ${ }^{1}$.

Para colaborar con los tomadores de decisión y las autoridades regulatorias de vacunas, la SLIPE y la Americas Health Foundation (AHF) tomaron la iniciativa de organizar una reunión para discutir temas relevantes vinculados al uso de la vacuna contra la poliomielitis en América Latina. Esta reunión fue realizada con un grupo de expertos de la Sociedad Latinoamericana de Infectología Pediátrica (SLIPE) (Polio Task Force Meeting), con la participación de representantes de los 
Programas Nacionales de Vacunación y/o de Sociedades Médicas de Pediatría de varios países de América Latina. Incluso, algunos países tuvieron representación de ambas entidades. También fueron invitados representantes de la OMS, de la Organización Panamericana de la Salud (OPS), de la Academia Americana de Pediatría (AAP) y otras autoridades de salud.

La propuesta de la SLIPE y la AHF fue discutir con representantes de las mencionadas entidades sus tendencias, disponibilidad de vacunas, dificultades de cambios en sus esquemas de vacunación a una vacuna más segura y evaluar cuáles son sus incertidumbres, aliadas a sus inseguridades.

Como resultado, la SLIPE, tomando en cuenta diferentes factores tales como: los datos epidemiológicos actuales, los eventos adversos de la vacuna oral atenuada (OPV) contra poliomielitis, la disponibilidad de otra vacuna inactivada (IPV) inyectable que no tiene el potencial de causar los eventos adversos graves de la vacuna oral (OPV), la eficacia y la efectividad de la IPV en varios países del mundo donde es utilizada hace varios años, el racional del cambio del esquema de vacunación en diferentes países de la América Latina; hace pública su recomendación del cambio a la vacuna inactivada para América Latina, a través del presente documento, enfatizando su posición.

\section{Objetivos}

- Revisar los Programas de Vacunación contra la polio en los países de América Latina.

- Analizar los mejores mecanismos para implementar la estrategia de la OMS de poner Punto Final a la polio a través del cambio de la vacuna oral de virus vivos atenuados (OPV) a la vacuna inactivada contra la polio (IPV).

- Definir el rol de las vacunas combinadas que contengan polio como una estrategia complementaria, para alcanzar altos niveles de cobertura de vacunación y así facilitar la implementación de IPV.

- Definir y preparar un documento llamado Declaración de la Sociedad Latinoamericana de Infectología Pediátrica (SLIPE) con un Plan de Acción para el período 2014-2015 con respecto a las políticas de vacunación contra la polio en Latinoamérica.

\section{Situación epidemiológica retrospectiva de la polio hasta la actualidad}

Desde 1988, cuando se estimaron 350.000 casos de polio en el mundo en 125 países, la OMS, a través de la Global Polio Eradication (GPE), los gobiernos, las sociedades civiles y comunidades alrededor del mundo, están involucrados y concentrando esfuerzos para erradicar esta enfermedad causada tanto por el poliovirus salvaje, como por el poliovirus derivado de la vacuna. Como resultado de los esfuerzos globales, el número de casos de polio salvaje disminuyó un 99\%, llegando a 406 casos en $2013^{2}$.

A la fecha, sólo hay tres países que permanecen con polio endémica, que en orden de acuerdo al número de casos registrados hasta fines de mayo de 2014, son: Pakistán: 66, Afganistán: 4 y Nigeria: 3. y algunos con casos importados de estos países en: Guinea Ecuatorial: 3, Camerún: 3, Irak: 1, Siria: 1, Etiopía: 1, totalizando 82 casos de polio salvaje serotipo $1^{3}$.

Por primera vez en la historia, todos los casos de polio causados por un virus salvaje desde 2013 se debieron a un solo serotipo, el tipo 1. El caso más reciente debido a un poliovirus salvaje tipo 3, ocurrió el 10 de noviembre de 2012 en Nigeria. El serotipo salvaje 2, no ha sido detectado desde 1999. El poliovirus circulante tipo 2 derivado de la vacuna sigue siendo actualmente el más importante agente causal de casos de poliomielitis, altamente concentrados en Pakistán y la frontera de Camerún, Chad, Níger y Nigeria ${ }^{4,5}$.

Mientras haya un niño infectado, otros niños de diferentes países están en riesgo de contraer la polio. El fracaso en erradicar la polio de estos países puede resultar en 200.000 nuevos casos por año en el mundo, dentro de los próximos 10 años ${ }^{6}$.

La polio salvaje fue eliminada de las Américas en 1991 y ésta fue la primera Región de la OMS, en obtener la Certificación de Erradicación de Polio (salvaje) en 1994. Según la OMS, la Región del Pacifico Occidental y Europa obtuvieron sus Certificados de Erradicación en 2000 y 2002, respectivamente.

Más recientemente, este año 2014, después del último caso registrado en India en 2011, la región del Sudeste Asiático, con 1,8 billones de personas, obtuvo su Certificado de Erradicación de Polio (salvaje).

Aunque los países estén firmemente comprometidos con el proceso de erradicación, según la OMS, Pakistán (en la región noroeste de la ciudad de Peshawar) es considerado actualmente el mayor reservorio de poliovirus endémico en el mundo, además de generar una enorme preocupación por la increíble violencia que hay contra los vacunadores. Líderes religiosos generan dificultades con las campañas de vacunación, diciendo, por ejemplo, que la vacuna contra la polio causa esterilidad en los niños musulmanes?.

\section{Vacunas contra polio, eventos adversos y sus consecuencias}

Una vez contraída la poliomielitis no hay cura para la enfermedad. Sólo puede ser prevenida mediante la vacunación. 
Actualmente hay dos tipos de vacunas disponibles para la prevención del poliovirus salvaje, las que son utilizadas para aplicación en el calendario de rutina en algunos países de América Latina (AL): la vacuna inactivada parenteral contra los poliovirus 1, 2 y 3 (IPV) y la vacuna atenuada oral trivalente contra los poliovirus 1, 2, 3 (tOPV, o conocida como OPV). Existen también vacunas sintetizadas para uso en la fase de transición, la vacuna oral atenuada bivalente contra el poliovirus 1, 3 (bOPV 1 y 3 ) y la vacuna oral monovalente contra el poliovirus 1 y 3 (mOPV1 y mOPV3), para control de brotes y para substituir la vacuna atenuada oral trivalente. Las vacunas bOPV 1-3 y mOPV1 y mOPV3 son equivalentes y generan mayor inmunidad que la tOPV1-2-3 .

Cuando la tOPV fue sintetizada en los años 60, reemplazó a la vacuna parenteral atenuada (IPV) que ya había sido sintetizada en 1957 y estaba siendo aplicada en territorio americano.

A pesar de ser una poderosa herramienta para prevenir la polio, ya que es una vacuna fácilmente administrada por vía oral y a precios relativamente bajos, la OPV tiene algunas desventajas que pueden representar un alto costo en la actualidad. Se sabe que actualmente es necesario aplicar varias dosis para alcanzar una eficacia aceptable. Aun así, niños con polio salvaje en India habían recibido 10 o más dosis de tOPV 9 .

Asimismo se pueden citar otras desventajas: además de tener un riesgo bajo de causar polio paralítica asociada a la vacunación (del inglés vaccine associated paralytic poliomyelitis-VAPP) entre los vacunados o sus contactos cercanos, se sabe actualmente que los virus de las vacunas pueden ser transmitidos a través de portadores humanos, y pueden experimentar mutaciones y revertir genéticamente hacia un tipo de virus salvaje de transmisión y virulencia, además de interactuar con otros enterovirus a nivel intestinal. El riesgo de VAPP en receptores de vacuna atenuada varía de acuerdo a los países de América Latina, desde 1 caso en 2,56 a 4,10 millones de recién nacidos ${ }^{10}$. Estas cifras pueden variar de acuerdo con la Vigilancia Epidemiológica (VE) de Parálisis Flácida de cada país.

Si este virus mutante derivado de la vacunación (VDPV) permanece circulando en la comunidad durante un año o más, puede causar divergencia genética de la cepa vacunal Sabin, en grados que pueden variar a partir de $1 \%$ al año. Los virus VDPV, reconocidos por su alta divergencia genética con respecto a las cepas de la vacuna oral contra la polio (OPV), pueden entrar en tres categorías: 1) cVDPVs de brotes, usualmente, pero no exclusivamente; 2) iVDPVs de pacientes con inmunodeficiencias primarias; y 3) VDPVs ambiguos (aVDPVs), de origen no identificado.

En el caso de que este virus circulante y mutante, cVDPV, alcance a la población susceptible, a causa por ejemplo, de una baja cobertura de vacunación, puede desencadenar brotes de poliomielitis, con cuadros de enfermedad semejantes a los provocados por virus salvajes. Los cVDPVs son biológicamente equivalentes a los poliovirus salvajes, surgen cuando las poblaciones tienen baja inmunidad específica para un serotipo y pueden circular indefinidamente. Los poliovirus derivados de la vacuna asociados a inmunodeficiencia (iVDPVs) continuarán emergiendo mientras se siga utilizando la OPV. Los iVDPVs pueden permanecer en circulación por largo tiempo, desapercibidos en el intestino de pacientes inmunosuprimidos, en el medio ambiente y en las alcantarillas (aVDPV), representando un riesgo enorme para la reintroducción de los virus en regiones en las que el virus ya fue erradicado ${ }^{11}$.

En general, un virus es definido como VDPV si tiene una divergencia $\geq 1 \%$ en la secuencia VP1 comparado con la cepa Sabin correspondiente ${ }^{12}$.

La enfermedad de polio causada por el virus mutante derivado de la vacuna atenuada tiene las mismas características clínicas y discapacitantes que la polio causada por el virus salvaje. En consecuencia, con los datos disponibles actualmente, continuar con la utilización de la vacuna atenuada representa un riesgo para la erradicación global de la polio.

En 2003, cuando hubo una reducción substancial del número de casos en todo el mundo, el sueño de que "la polio sería próximamente historia" parecía aproximarse.

Sin embargo, en 2001 fue reconocido el brote de Polio por cVDPV serotipo 1 en la Isla de Hispaniola (República Dominicana y Haití) con 21 casos oficialmente reportados. Luego, varios otros brotes por cVDPV fueron reportados en el mundo, además de que el virus del brote de Egipto en 1988 fue reconocido como causado por el poliovirus serotipo 2.

Datos aportados por la OMS con respecto a cVDPV suman, desde el año 2000 hasta marzo de 2014, más de 500 casos, provocados en su gran mayoría por el poliovirus serotipo $2^{3}$.

Es necesario resaltar que por cada caso reportado se estima que hay de 100 a 1.000 infectados, diseminando poliovirus por el medio ambiente ${ }^{12}$.

\section{Vigillancia epidemiológica de parálisis flácida aguda y vigillancia ambiental}

A nivel país, la vigilancia de la parálisis flácida aguda (PFA) es el estándar de excelencia para detectar casos de poliomielitis. Hay una gran dificultad para obtener datos de vigilancia epidemiológica de PFA en los diversos países de América Latina, alcanzando apenas, los índices recomendados por la OMS para esta región del mundo. Algunos países no los alcanzan.

En las regiones endémicas, para asegurar una alta 
sensibilidad en la vigilancia epidemiológica de PFA, la tasa mínima de PFA es de 2/100.000 habitantes en $\leq 15$ años. En las Américas es de 1/100.000 habitantes. Si se tiene en cuenta la cifra más alta recomendada para otras regiones para lograr mayor sensibilidad, entonces, prácticamente toda América Latina quedaría por debajo de los indicadores de vigilancia epidemiológica para PFA, permitiendo la detección de los casos de cVDPV y VAPP. Otro parámetro a considerar, es la calidad de la notificación (el envío de muestras de heces de manera adecuada) que demuestra ser mucho menor que la recomendación de $80 \%$, en toda América Latina ${ }^{13}$.

Por lo tanto, se puede deducir que la sub-notificación es evidente, especialmente en los casos de VAPP.

Aunque haya fallas en la VE, sabemos que algunos países tienen reportados casos de VAPP, con divergencia genética variable, aunque la publicación rutinaria de estos casos no siempre ocurra ${ }^{10}$.

Otra falla de notificación que debemos recordar es la de Vigilancia Ambiental (VA) para la detección de los poliovirus aVDPV ( in fuente conocida) en las alcantarillas. A pesar de que la VA sea cara, es realizada por algunos países. La VA incluye el control de aguas residuales $\mathrm{u}$ otras muestras ambientales para verificar la presencia de poliovirus. Adicionalmente, la VA frecuentemente confirma infecciones por poliovirus salvaje en ausencia de casos de parálisis y complementa la vigilancia de $\mathrm{PFA}^{13,14}$.

Por todos los motivos expresados anteriormente, especialmente los referidos a los eventos adversos de la vacuna atenuada, la OMS y otras autoridades de salud están de acuerdo con que la circulación del virus atenuado debe ser interrumpida. Para lograrlo, la vacuna atenuada deberá ser discontinuada lo más pronto posible, en una actividad coordinada entre los diversos países que todavía siguen utilizando esta vacuna. La recomendación de la OMS es que en este momento de transición hasta 2015, por lo menos una dosis de IPV deba ser incluida en los calendarios de vacunación de rutina de los niños, hasta lograr la discontinuación definitiva de la vacuna de virus vivos atenuados ${ }^{4,15}$.

La decisión de la OMS con respecto a la erradicación global de la poliomielitis, o sea, tanto de la enfermedad provocada por el poliovirus salvaje, como la provocada por el virus vacunal, debe lograrse mediante la asociación de recursos públicos y privados, además de los reconocidos esfuerzos de las comunidades en los países en los cuales la polio sigue siendo endémica. Según la OMS, en este momento no es posible retroceder. Mantener la situación tal como está, sólo intentando controlar la polio y no erradicarla es mucho más difícil y costoso que su erradicación, sin considerar los aspectos de inequidad e injusticia social.

Transcurridos 23 años de la erradicación de la polio en las Américas, los países de este continente se encuentran frente a una situación paradójica: seguir con la vacuna atenuada y tener la enfermedad causada por el virus vacunal, o cambiar a una vacuna inactivada, más segura y efectiva. Es por este motivo que en este momento, la decisión de la OMS es brindar apoyo para la toma de una posición más confiable y segura de los Programas Nacionales de Inmunización de América Latina.

Aunque el poliovirus serotipo 2 no haya sido aislado desde 1999, sigue siendo la causa de la gran mayoría de los casos de cVDPV. Entonces el proceso de transición incluye una etapa intermedia que es la sustitución de la vacuna tOPV por una vacuna de mayor eficacia y poder inmunogénico, que es la vacuna bivalente atenuada bOPV 1 y 3 , disponible desde hace algunos años, bastante más eficaz que la tOPV con el mismo número de dosis ${ }^{16}$.

El racional para la introducción de por lo menos una dosis de IPV, en cualquier momento de la inmunización del niño, previo a la suspensión de OPV2 es: *la posibilidad de la continua circulación silenciosa del cVDPV2 y de una nueva emergencia de un cVDPV2 luego del cese de la OPV2; *un riesgo real de brotes por VDPV2 luego del cese de OPV2, debido a una falla en el control, de excretores crónicos de VDPVs.

Los beneficios de una dosis de IPV previo al cese de OPV son: prevenir la polio en caso de exposición al VDPV2 o al WPV2; mejorar la respuesta al mOPV2 en caso de un brote; reducir la transmisión de un poliovirus tipo 2 reintroducido y reforzar la inmunidad a los poliovirus salvajes 1 y $3^{17,18}$.

En este proceso de cambio de la vacuna atenuada hacia la vacuna inactivada, algunos países ya tienen incluida la IPV en sus calendarios, ya sea en un esquema secuencial (IPV/OPV) o completo con IPV en todas las dosis. En 2012, América Latina y el Caribe, con más de 10 millones de nacimientos al año, ya tenían más de $50 \%$ de su población bajo régimen IPV (ej, Brasil, Uruguay, Costa Rica, México), a nivel público y privado, como IPV sola o combinada a otros antígenos ${ }^{18-21}$.

Algunos países, independientemente de sus esquemas primarios, todavía siguen utilizando campañas anuales de vacunación con OPV (Brasil, México y Costa Rica), otros no, pero realizan refuerzos con la vacuna OPV.

Los países que todavía continúan aplicando la vacuna OPV tienen que prepararse para este cambio de tOPV a bOPV en sus programas nacionales de vacunación y, en poco tiempo, hacia IPV. Esta medida, además de ser segura tiene la ventaja de permitir el gradual aprovisionamiento de la vacuna IPV, manteniendo la sustentabilidad de los programas de inmunización.

Se debe mencionar que la cobertura de vacunación es alta en todos los países de América Latina, aunque haya sectores de baja cobertura. Esto es reconocido y debe ser solucionado mediante una posición firme de los gobiernos $^{22,23}$. 


\section{Conclusiones y recomendaciones del Taller de SLIPE (Polio/2014)}

\section{Esquema con IPV}

- Según la SLIPE, el esquema óptimo propuesto son cuatro dosis de IPV (tres dosis en el esquema primario más un refuerzo), ya sea IPV combinada o no combinada con otras vacunas indicadas en el programa de inmunización del país. Es aceptable y no hay prejuicio de la respuesta inmunológica, de que el niño vacunado con vacunas combinadas reciba más dosis de IPV que las recomendadas en el esquema primario.

- Durante la etapa de transición de la vacuna OPV a la IPV se debe tener en consideración que:

a) Se puede optar por un esquema que combine las $2 \mathrm{o}$ las 3 primeras dosis de IPV seguidas por una o dos dosis de OPV.

b) La dosis de los 4 años se puede dar con IPV o con OPV. Esta última puede administrarse en el esquema de rutina o mediante estrategias masivas durante los Días Nacionales de Vacunación.

- Niños vacunados con OPV, independientemente de su esquema anterior, deben completar su inmunización contra la poliomielitis con la vacuna IPV.

- La vacuna IPV puede ser aplicada en presentación mono componente o en forma de vacunas combinadas en cualquiera de las dosis.

- Niños correcta y completamente vacunados con la vacuna IPV no necesitan dosis posteriores de vacuna contra polio para su protección individual.

- Cualquier esquema que tenga OPV, independientemente de que sea tri, bi o monovalente, deberá ser discontinuado posteriormente para ser sustituido por un esquema completo con IPV, dado que si la vacuna atenuada sigue siendo administrada el virus seguirá circulando.

\section{Coberturas}

- Los países deben plantear las estrategias óptimas para mantener y mejorar las coberturas de vacunación.

- Disminuir los sectores de baja cobertura existentes en varios países, incluyendo poblaciones desplazadas.

- Implementar el registro nominal de vacunas.

\section{Vigilancia epidemiológica y ambiental}

- Mejorar la vigilancia epidemiológica de la Parálisis Flácida Aguda (PFA) de acuerdo a los criterios establecidos por la OMS.

- Establecer un programa de vigilancia ambiental para obtener datos de la circulación del virus.
Estrategias para la introducción de IPV en los Programas Nacionales de Inmunización

- Establecer una comunicación adecuada con la población, que reúna los siguientes elementos:

- Utilizar un lenguaje de fácil entendimiento para todos los niveles sociales.

- Facilitar la comunicación con la prensa (incluida la prensa escrita) y otros medios de comunicación.

- Optimizar el uso de redes sociales como una herramienta para diseminar los mensajes, sobre todo con la juventud.

- Solicitar el apoyo de sociedades científicas en las comunicaciones para establecer mayor credibilidad.

- Enfocar la comunicación en mantener la confianza de la población en los programas de vacunación, incluyendo la polio.

- Alinear los mensajes de las sociedades científicas y de los ministerios de salud de los países.

- Acercamiento de las sociedades científicas a los tomadores de decisión en los ministerios y a las sociedades civiles en los países.

- En el caso del acercamiento a los tomadores de decisión, las sociedades científicas pueden formar parte de comités asesores u otro tipo de grupos de expertos.

- Con respecto a las sociedades civiles, se puede brindar un importante apoyo científico.

- SLIPE, como sociedad internacional, debe seguir colaborando con las sociedades científicas de los países que soliciten su apoyo.

- Solicitar formalmente la colaboración de la OPS con la SLIPE, en preparación para la introducción de IPV a través de:

- Un apoyo más técnico/académico en el tema.

- Un mayor acercamiento con las sociedades científicas de los países.

- La inclusión de expertos de SLIPE como parte del TAG.

- Asegurar el abastecimiento y precios óptimos para la introducción de IPV por parte de los proveedores de vacunas para garantizar la sustentabilidad del programa.

- Programarse para incorporar IPV (y discontinuar OPV) y de esta manera anticipar las nuevas demandas e iniciar con tiempo la comunicación de los requerimientos de IPV a los proveedores.

- Exhortar a los proveedores una comunicación temprana de cualquier limitación en el abastecimiento de la vacuna que pudiera surgir.

- Negociar precios que sean factibles para la introducción de IPV tanto en forma combinada o monocomponente. 
- Capacitar a los vacunadores como fuente importante de información e implementación de los programas.

- Se recomienda comenzar por la capacitación de aquellos con mayor experiencia, conocimiento y liderazgo para que luego ellos colaboren para capacitar al resto.

- Mejorar la logística de distribución y almacenamiento de vacunas, especialmente lo que respecta al mantenimiento de la cadena de frío.

\section{Consideraciones finales}

Estamos a un paso de la erradicación de la polio. No es justo para los niños que no se entienda esta necesidad y que se siga utilizando una vacuna que por sí misma puede provocar la enfermedad que se está intentando combatir.

Aparte de la evidencia científica, los países que todavía no han decidido hacer el cambio a IPV deben considerar las implicaciones del punto anterior por cuestiones de equidad y justicia social.

Es importante aclarar que el esquema propuesto por la SLIPE es una recomendación y que los países tomarán las decisiones de acuerdo a sus necesidades. Obviamente cada país debe tener un presupuesto destinado para los programas de inmunización, incluyendo la introducción de nuevas vacunas, y la sustentabilidad de dichos programas. También hay que recordar que cada Día de Vacunación con la vacuna oral atenuada cuesta millones de dólares y la conocida pérdida de vacuna oral es muy grande lo que eleva mucho el costo (para administrar una dosis se pierden varias dosis).

En este proceso hay desafíos, retos y dificultades. El objetivo de esta reunión fue contribuir a evaluar el cambio a IPV, compartiendo experiencias y permitiendo el intercambio de ideas entre países que cambiaron sus esquemas con países que todavía no lo han hecho.

En este momento, es fundamental la colaboración de todos en este camino hacia la erradicación de la polio, enfermedad causada tanto por el virus salvaje como por el virus vacunal.

Cabe resaltar la recomendación de la OMS, que para el 2015 todos los países deben haber incluido por lo menos una dosis de IPV en sus programas de vacunación. Además, los países necesitan programarse para suspender OPV y continuar con el uso de IPV, ya sea en su presentación monocomponente o combinada.

¡Solamente con la erradicación de la polio, podemos terminar con la inequidad y promover la justicia social!
Agradecimientos. A todos los participantes a la reunión, quienes representando a sus entidades colaboraron y contribuyeron con su experiencia y sus opiniones para lograr desarrollar esta declaración:

- Miembros de la SLIPE expertos en polio de Argentina, Brasil, Colombia, Costa Rica, Estados Unidos, México y República Dominicana;

- Miembros del Programa Ampliado de Inmunizaciones de: Argentina, Brasil, Chile, El Salvador, Honduras, México, Panamá, Paraguay, Perú y Uruguay; representación de la Academia Americana de Pediatría.

El auspicio de la Americas Health Foundation-AHF (Washington DC, Estados Unidos).

A la Sra. Herlys Gianelli por su colaboración durante la reunión, por la compilación de la información y la redacción de las conclusiones y recomendaciones.

A la Sra. Valeria Castro por su contribución a la revisión de la redacción del documento.

\section{Resumen}

A pesar de que ya se ha recorrido el $99 \%$ del camino para erradicar la poliomielitis (polio) del mundo, esta enfermedad continúa causando parálisis en niños. $\mathrm{Su}$ erradicación no sólo significa el fin de la circulación del poliovirus salvaje, sino también del virus derivado de la vacunación. La Sociedad Latinoamericana de Infectología Pediátrica (SLIPE), tomando en cuenta diferentes factores tales como: los datos epidemiológicos actuales, los eventos adversos de la vacuna oral atenuada (OPV) contra polio, la disponibilidad de una vacuna inactivada (IPV) inyectable que no tiene el potencial de causar los eventos adversos graves de la OPV, la eficacia y la efectividad de la IPV en varios países del mundo donde es utilizada desde hace varios años, el racional del cambio del esquema de vacunación en diferentes países de la América Latina; hace pública su recomendación del cambio a la IPV para América Latina, a través de la presente Declaración con un Plan de Acción para el período 2014-2015 con respecto a las políticas de vacunación contra la polio en Latinoamérica. 1. El esquema óptimo propuesto son cuatro dosis de IPV (tres dosis en el esquema primario más un refuerzo), ya sea IPV combinada o no combinada con otras vacunas indicadas en el programa de inmunización del país. Es aceptable un esquema alternativo durante la etapa de transición de la vacuna OPV a la IPV; 2. Los países deben plantear las estrategias óptimas para mantener y mejorar las coberturas de vacunación e implementar el registro nominal de vacunas; 3 . Mejorar la vigilancia epidemiológica de la Parálisis Flácida Aguda (PFA) y establecer un programa de vigilancia ambiental; 4. Establecer estrategias para 
la introducción de IPV en los Programas Nacionales de Inmunización, como comunicación adecuada con la población y otras; 5 . Acercamiento de las sociedades científicas a los tomadores de decisión; 6. Asegurar el abastecimiento y precios óptimos para la introducción de la IPV; 7. Capacitar a los vacunadores; 8 . Mejorar la logística de distribución y almacenamiento de vacunas. Además de la evidencia científica, los países que todavía no han decidido hacer el cambio a IPV deben considerar las implicaciones de equidad y justicia social.

\section{Referencias bibliográficas}

1.- WHO: Ten facts on Polio eradication. http:// www.who.int/features/factfiles/polio/en/. Available 26-04-14

2.- WHO-Poliomyelitis: intensification of the global eradication initiative-Report by the Secretariat. Sixty Seventh World Health Assembly-March 21, 2014.

3.- Global Polio eradication Initiative. Wild Polio Cases. http://www.polioeradication. org/Dataandmonitoring/Poliothisweek.aspx. (Consultado el 25 de mayo de 2014).

4.- World Health Organization-Poliomyelitis. http:// apps.who.int/gb/ebwha/pdf_files/WHA67/ A67_38-en.pdf. (Consultado el 19 de abril de 2014).

5.- Global Polio eradication Initiative. Polio. Data and Monitoring. (http://www.polioeradication. org/Dataandmonitoring.aspx). (Consultado el 19 de abril de 2014).

6.- World Health Organization. Polio eradication. Fact Sheets número 114. http://www.wh.int/ mediacentre/factsheets/fs114/en/ (Consultado el 19 de abril de 2014).

7.- Pakistani City Is World's Biggest Reservoir of Polio Viruses. Medscape. Jan 17, 2014).

8.- Sutter R W, John T J, Jain H, Agarkhedkar S, Ramanan P V, et al. Immunogenicity of bivalent types 1 and 3 oral poliovirus vaccine: a randomized, double-blind, controlled trial. Lancet 2010 nov 13; 376 (9753): 1682-8. doi: 10.1016/S0140-6736(10)61230-5. Epub 2010 oct 25 .

9.- Paul Y. Polio eradication: experts have misled us. Medical Veritas 3 (2006) 781-5.
10.- Landaverde J M, Trumbo S P, DanovaroHolliday M C, Cochi S E, Gandhi R, et al. Vaccine-associated paralytic poliomyelitis in the post elimination era in Latin America and the Caribbean, 1992-2011. J Infect Dis 2014 may; 209 (9): 1393-402. doi: 10.1093/infdis/jit602. Epub 2014 feb 11.

11.- Centers for Disease Control and Prevention. MMWR Morb Mortal Wkly Rep July 1 , 2011; 60: 846-50. Update on VaccineDerived Polioviruses-Worldwide, April 2011-June 2012.

12.- Centers for Disease Control and Prevention. MMWR Morb Mortal Wkly Rep. Update on Vaccine-Derived Polioviruses-Worldwide, April 2011-June 2012. 2012; Sept 21, 61 (37); 741-6.

13.- Wringe A, Fine PE, Sutter RW, Kew OM. Estimating the extent of vaccine-derived poliovirus infection. PLOSOne. 2008; 3 (10): e3433. doi: 10.1371/journal.pone. 0003433 . Epub 2008 Oct 29

14.- Global Polio Eradication Initiative. Acute Flaccid Paralysis (AFP) surveillance. Consultado: http://www.polioeradication. org/Dataandmonitoring/Surveillance.aspx (Consultado el 21 de mayo de 2014).

15.- Global Polio eradication Initiative. Update on Vaccine-Derived Polioviruses-Worldwide, April 2011-June 2012 Weekly September 21, 2012/61(37); 741-6.

16.- World Health Organization. Polioeradication http://www.polioeradication.org/ Resourcelibrary/Strategyandwork/ EmergencyActionPlan.aspx. (Consultado el 19 de abril de 2014).
17.- WHO-Sixty-Seventh World Health Assembly-Poliomyelitis: intensification of the global eradication initiative - 21 March 2014.

18.- WHO-SAGE - Global Polio eradication Initiative-Meeting November 2012. http://www. who.int/immunization/sage/meetings/2012/ november/Yellow_book_SAGE_Nov_2012 final.pdf?ua $=1$. (Consultado el 26 de abril de 2014)

19.- Source: Population Reference Bureau, 2011 http://www.prb.org/pdf11/2011 population-datasheet_sp.pdf.

20.- Ministerio da Saude do Brasil. Vacinaçao. http:// www.brasil.gov.br/saude/2009/12/campanhasde-vacinacao-2. (Consultado el 25 de mayo de 2014).

21.- Ministerio de la Salud de Costa Rica. Vigilancia de Salud. http://www.ministeriodesalud. go.cr/index.php/vigilancia-de-la-salud/iniciovigilancia-normas-protocolos-guias-ms. (Consultado el 20 de mayo de 2014).

22.- Ministerio de la Salud de México. Esquemas de Vacunación. http://censia.salud.gob.mx/ contenidos/vacunas/esquemavacunas.html (Consultado el 20 de mayo de 2014).

23.- WHO/PAHO-Immunization in the Americas2013-Summary. Comprehensive Family Immunization Family, Gender and Life Course. (Consultado el 27 de abril de 2014).

24.- WHO PAHO-Health Situation in The Americas: Basic Health Indicators 2013. http://www. paho.org/hq/index.php?option=com_co ntent\&view $=$ article $\&$ id $=2470 \% 3$ Adatastatistics\&catid $=1900 \% 3$ Adata-statisticshome\&Itemid $=2003 \&$ lang $=p t$ 


\title{
The challenge of changing the inactivated poliomyelitis vaccine in Latin America. Declaration of the Latin American Society of Pediatric Infectious Diseases (SLIPE)
}

\author{
Luiza Helena Falleiros-Arlant, María Luisa Avila Agüero, José Brea del Castillo and Cristina Mariño
}

\section{El desafío del cambio de la vacuna inactivada contra poliomielitis en América Latina. Declaración de la Sociedad Latinoamericana de Infectología Pediátrica (SLIPE)}

A pesar de que ya se ha recorrido el 99\% del camino para erradicar la poliomielitis (polio) del mundo, esta enfermedad continúa causando parálisis en niños. Su erradicación no sólo significa el fin de la circulación del poliovirus salvaje, sino también del virus derivado de la vacunación. La Sociedad Latinoamericana de Infectología Pediátrica (SLIPE), tomando en cuenta diferentes factores tales como: los datos epidemiológicos actuales, los eventos adversos de la vacuna oral atenuada (OPV) contra polio, la disponibilidad de una vacuna inactivada (IPV) inyectable que no tiene el potencial de causar los eventos adversos graves de la OPV, la eficacia y la efectividad de la IPV en varios países del mundo donde es utilizada desde hace varios años, el racional del cambio del esquema de vacunación en diferentes países de la América Latina; hace pública su recomendación del cambio a la IPV para América Latina, a través de la presente Declaración con un Plan de Acción para el período 2014-2015 con respecto a las políticas de vacunación contra la polio en Latinoamérica. 1. El esquema óptimo propuesto son cuatro dosis de IPV (tres dosis en el esquema primario más un refuerzo), ya sea IPV combinada o no combinada con otras vacunas indicadas en el programa de inmunización del país. Es aceptable un esquema alternativo durante la etapa de transición de la vacuna OPV a la IPV; 2. Los países deben plantear las estrategias óptimas para mantener y mejorar las coberturas de vacunación e implementar el registro nominal de vacunas; 3 . Mejorar la vigilancia epidemiológica de la Parálisis Flácida Aguda (PFA) y establecer un programa de vigilancia ambiental; 4. Establecer estrategias para la introducción de IPV en los Programas Nacionales de Inmunización, como comunicación adecuada con la población y otras; 5 . Acercamiento de las sociedades científicas a los tomadores de decisión; 6 . Asegurar el abastecimiento y precios óptimos para la introducción de la IPV; 7. Capacitar a los vacunadores; 8. Mejorar la logística de distribución y almacenamiento de vacunas. Además de la evidencia científica, los países que todavía no han decidido hacer el cambio a IPV deben considerar las implicaciones de equidad y justicia social.

Key words: Poliomyelitis, Polio vaccines, Polio vaccination schedules, Attenuated polio vaccine (OPV), Inactivated polio vaccine (IPV), Polio in Latin America.

Palabras clave: Poliomielitis, Vacunas contra la polio, Esquema de vacunación contra la polio, Vacuna atenuada anti-polio (OPV), Vacuna inativada anti-polio (IPV), Polio en América Latina, Erradicación de la polio, Vacuna oral contra la polio.

\section{Introduction}

$\mathrm{P}$ oliomyelitis (polio) is still causing paralysis in children. Unlike most diseases, polio can be completely eradicated. We have already covered $99 \%$ of the path to eradicate it from the world. The eradication of this disease means the end of all poliovirus circulation, both wild poliovirus and vaccine-derived poliovirus.

In 2014 , there are only three countries where polio transmission has not been stopped yet. We must become aware of this historical opportunity to win this battle. The World Health Organization (WHO) has low-cost and efficacious vaccines to prevent polio. We should remain focused and determined, since we have the tools and technical knowledge to eradicate the virus ${ }^{1}$.

In order to collaborate with decision makers and regulatory authorities for vaccines, SLIPE and the Americas Health Foundation (AHF) took the initiative to organize a meeting to discuss relevant issues regarding the use of poliomyelitis vaccine in Latin America. This meeting included a group of experts from the Latin American Society of Pediatric Infectious Diseases (SLIPE) (Polio Task Force Meeting), with the participation of representatives of National Inmunization Programs and/or Pediatric 
Societies from several Latin American countries. Some countries even had representation of both entities. Representatives of WHO, Pan-American Health Organization (PAHO), American Academy of Pediatrics (AAP) and other health authorities were also invited.

SLIPE and AHF's proposal was to discuss with the representatives of the abovementioned authorities their trends, the availability of vaccines and the difficulties in changing their vaccination schedules to a safer vaccine, and to evaluate their uncertainties together with their insecurities.

As a result, taking into account different factors, such as: current epidemiological data, adverse events of the attenuated oral poliomyelitis vaccine (OPV), the availability of another injectable inactivated vaccine (IPV) without the potential of causing the severe adverse events of the oral vaccine (OPV), the efficacy and effectiveness of the IPV in several countries of the world where it has been used for several years, the rationale of changing the vaccination schedule in different Latin American countries; SLIPE, hereby, announces its recommendation of switching to the inactivated vaccine in Latin America, emphasizing its position.

\section{Objectives}

- Reviewing Polio Vaccination Programs in Latin American countries.

- Analyzing the best mechanisms to implement WHO's polio endgame strategy by switching from the liveattenuated virus oral vaccine (OPV) to the inactivated polio vaccine (IPV).

- Defining the role of combination vaccines containing polio as a complementary strategy, to reach high vaccination coverage levels, thus facilitating IPV implementation.

- Defining and preparing a document called Declaration of the Latin American Society of Pediatric Infectious Diseases (SLIPE) with an Action Plan for 2014-2015 period as regards polio vaccination against polio policies en Latin America.

\section{Polio's retrospective epidemiological situation to date}

Since 1988, when 350,000 cases of polio were estimated in 125 countries of the world, WHO, through the Global Polio Eradication (GPE), governments, civil societies and communities around the world have committed and concentrated their efforts to eradicate this disease, caused both by wild poliovirus and vaccine-derived poliovirus. As a result of the global efforts, the number of wild polio cases decreased by $99 \%$, dropping to 416 cases in $2013^{2,3}$.
To date, only three countries remain polio-endemic which, ordered by the number of cases recorded until August $17^{\text {th }}$ 2014, are: Pakistan: 180, Afghanistan: 8, and Nigeria: 5 . And some with cases imported from these countries in: Somalia: 4; Equatorial Guinea: 5, Cameroon: 5, Iraq: 2, Syria: 1, Ethiopia: 1, adding up to 139 cases of wild polio serotype $1^{3}$.

For the first time in history, in 2013 all cases of polio caused by a wild virus were due to a single serotype, type 1. The most recent case due to a wild poliovirus type 3 occurred on November $10^{\text {th }}, 2012$, in Nigeria. Wild serotype 2 has not been detected since 1999. The circulating vaccine-derived poliovirus type 2 continues to be the most important causal agent of cases of poliomyelitis, heavily concentrated in Pakistan and the border area of Cameroon, Chad, Niger and Nigeria ${ }^{4,5}$.

As long as a single child is infected, other children in different countries are at risk of contracting polio. Failure to eradicate polio from these countries could result in 200,000 new cases every year, within 10 years, all over the world ${ }^{6}$.

Wild polio was eradicated from The Americas in 1991, and this was the first WHO Region to achieve the Certification of (Wild) Polio Eradication in 1994. According to WHO, the Western Pacific Region and European Region achieved their Certification of Eradication in 2000 and 2002, respectively.

More recently, this year 2014, after the last case reported in India in 2011, the South-East Asian region, with 1.8 billion people, achieved the Certification of (Wild) Polio Eradication.

Although countries are resolutely committed to the eradication process, according to WHO, Pakistan (in the northwestern region of the city of Peshawar) is currently considered the largest reservoir of endemic polio viruses in the world, besides generating a strong concern over the incredible violence against vaccination teams. Some religious leaders generate difficulties with the vaccination campaigns, saying, for instance, that the polio vaccine causes sterility in Muslim children?

\section{Polio vaccines, adverse events and their consequences}

Once poliomyelitis is contracted, there's no cure for this disease. It can only be prevented through immunization.

Currently, there are two types of vaccines available to prevent wild poliovirus, which are used in the routine schedule in some Latin American (LA) countries: the inactivated parenteral poliovirus vaccine (IPV) types 1, 2 and 3 and the trivalent attenuated oral poliovirus vaccine (tOPV, also known as OPV) types 1, 2 and 3. There are 
also synthesized vaccines for the transitional phase, the bivalent attenuated oral poliovirus vaccine types 1 and 3 (bOPV 1 and 3) and the monovalent oral poliovirus vaccine types 1 and 3 (mOPV1 and mOPV3), to control outbreaks and to substitute the trivalent attenuated oral vaccine. The bOPV 1-3 and mOPV1 and mOPV3 vaccines are equivalent and provide higher immunity than tOPV1-2-38.

When tOPV was synthesized in the 1960s, it replaced the attenuated parenteral vaccine (IPV), which had already been synthesized in 1957 and was being administered in the American continent.

Despite of being a powerful tool to prevent polio, since it is an oral vaccine, easy to administer and at relatively low prices, the OPV has some disadvantages that nowadays can represent a high cost. It is known that, currently, several doses are necessary to achieve acceptable efficacy. Even so, children who contracted wild polio in India had received 10 or more tOPV doses ${ }^{9}$.

Other disadvantages can also be mentioned: in addition to presenting a low risk of causing vaccine associated paralytic poliomyelitis (VAPP) among the vaccinees or their close contacts, vaccine viruses are currently known to be able to be transmitted through human carriers and to experience mutations and revert to a wild virus type of transmission and virulence, as well as interacting with other enterovirus in the intestine. The risk of VAPP in attenuated vaccine recipients varies from country to country in Latin America, from 1 case per 2.56 to 4.10 million of newborns ${ }^{10}$. These figures may vary according to the Epidemiological Surveillance of Flaccid Paralysis of each country.

If this vaccine-derived mutant virus (VDPV) continues circulating in the community for a year or longer, it could cause genetic divergence of Sabin vaccine strain in degrees that can vary as of $1 \%$ per year. VDPV viruses, recognized by their high genetic divergence from the oral polio vaccine (OPV) strains, fall into three categories: 1) cVDPVs from outbreaks, usually, but not exclusively, 2) iVDPVs from patients with primary immunodeficiency, and 3) ambiguous VDPVs (aVDPVs), from an unidentified origin.

In case this circulating and mutant virus, cVDPV, reaches the susceptible population due to, for instance, low vaccination coverage, it may result in poliomyelitis outbreaks, with similar symptoms to the ones caused by wild viruses. cVDPVs are biologically equivalent to wild polioviruses, emerge when populations have lowserotype specific immunity and can circulate indefinitely. Immunodeficiency-associated vaccine-derived polioviruses (iVDPVs) will continue to emerge as long as OPV is used. iVDPVs can remain circulating unnoticed for a long time in the immunosuppressed patients' intestines, the environment and the sewers (aVDPV), representing a huge risk for reintroducing viruses in regions where the virus has already been eradicated ${ }^{11}$.

In general, a virus is defined as VDPV if it is $\geq 1 \%$ divergent in VP1 sequence compared to the corresponding Sabin strain ${ }^{12}$.

Polio disease caused by the attenuated vaccinederived mutant virus has the same clinical and crippling characteristics as polio caused by the wild virus. As a consequence, with the currently available data, continuing to use the attenuated vaccine represents a risk to global polio eradication.

In 2003, when there was a significant reduction of the number of cases in the entire world, the dream of "polio would soon be history" seemed to be close.

However, in 2001, an outbreak of polio caused by serotype $1 \mathrm{cVDPV}$ was recognized in Hispaniola (Dominican Republic and Haiti) with 21 officially reported cases. Afterwards, several other outbreaks due to cVDPV were reported in the world and, furthermore, the virus of the outbreak in Egypt, in 1988, was recognized as being caused by poliovirus serotype 2 .

According to data provided by $\mathrm{WHO}$, from the year 2000 to March 2014, there were more than 500 cases of cVDPV, caused mostly by poliovirus serotype $2^{3}$.

It should be emphasized that, for every reported case, an estimated 100 to 1000 infected people are spreading polioviruses in the environment ${ }^{12}$.

\section{Epidemiological surveillance of acute flaccid paralysis and environmental surveillance}

Nationwide, acute flaccid paralysis (AFP) surveillance is the gold standard for detecting cases of poliomyelitis. There's great difficulty in getting epidemiological surveillance data of AFP in Latin American countries, barely reaching the recommended indexes by WHO for this region of the world. Some countries don't achieve them.

In endemic regions, in order to ensure high sensitivity in AFP epidemiological surveillance, the minimum AFP rate is $2 / 100.000$ inhabitants in $\leq 15$ years. In The Americas, it is $1 / 100.000$ inhabitants. If the highest recommended figure for other regions to achieve higher sensitivity is taken into account, then practically all Latin America would be below the epidemiological surveillance indicators for AFP, allowing the detection of cVDPV and VAPP cases. Another parameter to be considered is the quality of reporting (adequate sending of stool samples), which shows to be much lower than the recommended $80 \%$ in all Latin America ${ }^{13}$.

Therefore, it can be deduced that sub-notification is evident, especially in VAPP cases.

Although epidemiological surveillance may present 
failures, we know that some countries have reported VAPP cases with variable genetics, though these cases may not be routinely published ${ }^{10}$.

Another notification failure to be remembered is that of environmental surveillance for aVDPV poliovirus detection (with no known source) in sewers. Although environmental surveillance is expensive, it is performed by some countries. Environmental surveillance includes testing sewage or other environmental samples for the presence of poliovirus. Additionally, environmental surveillance often confirms wild poliovirus infections in the absence of cases of paralysis and complements AFP surveillance ${ }^{13,14}$.

For all the reasons above mentioned, especially those referred to the adverse events of the attenuated vaccine, WHO and other health authorities agree that the circulation of the attenuated virus should be interrupted. To do so, the attenuated vaccine should be discontinued as soon as possible, in a coordinated activity among the different countries that are still using this vaccine. WHO recommends that, in this transition period until 2015, at least one IPV dose should be included in routine vaccination schedules for children, till the live-attenuated virus vaccine is definitely discontinued ${ }^{4,15}$.

WHO's decision as regards global poliomyelitis eradication, that is, the disease caused by wild poliovirus as well as the one caused by the vaccine virus, should be achieved by combining public and private resources, in addition to the recognized efforts of communities in countries where polio is still endemic. According to WHO, now it's not possible to go back. Keeping the situation just as it is, only trying to control polio and not eradicating it, is much harder and more expensive than eradicating it, not to mention inequity and social injustice aspects.

Twenty-three years after polio has been eradicated from The Americas, the countries of this continent face a paradoxical situation: whether to continue with the attenuated vaccine and have the disease caused by the vaccine virus or to switch to an inactivated, safer and more effective vaccine. This is why, currently, WHO's decision is to support the adoption of a more reliable and safer position for the National Immunization Programs of Latin America.

Even though poliovirus serotype 2 has not been isolated since 1999, it's still the cause of most of cVDPV cases. So, the transition process includes an intermediate phase that consists of replacing tOPV vaccine with a more efficacious vaccine with a higher immunogenic power: the bivalent attenuated bOPV 1 and 3 vaccine. This vaccine has been available for several years and is far more effective than tOPV with the same number of doses ${ }^{16}$.

The rationale for introducing at least one IPV dose, at any moment of the child's immunization, before suspending OPV2 is: *the possibility of the continuous cVDPV2 silent circulation and a new emergence of a cVDPV2 following OPV2 cessation; * a real risk of VDPV2 outbreaks following OPV2 cessation, due to containment failures, of chronic VDPV excretors.

The benefits of one IPV dose prior to OPV cessation are: preventing polio in case of being exposed to VDPV2 or WPV2; improving the response to MOPV2 in case of an outbreak; reducing the transmission of a reintroduced poliovirus type 2 , and boosting immunity to wild polioviruses type 1 and $3^{17,18}$.

In this process of switching from the attenuated vaccine to the inactivated vaccine, some countries have already included IPV in their schedules, whether it is in a sequential schedule (IPV/OPV) or in a complete one with IPV in every dose. In 2012, with more than 10 million births per year, Latin America and the Caribbean already had more than $50 \%$ of the population under IPV schedule (e.g., Brazil, Uruguay, Costa Rica, Mexico), at public and private levels, as IPV alone or combined with other antigens ${ }^{18-21}$.

Regardless of their primary schedules, some countries are still using annual vaccination campaigns with OPV (Brazil, Mexico and Costa Rica); others are not, but they administer booster doses of OPV.

Countries that are still administering OPV should get ready to switch from tOPV to bOPV and, soon, to IPV in their national immunization programs. In addition to being safe, this measure has the advantage of allowing the gradual IPV provisioning, keeping the sustainability of immunization programs.

It should be mentioned that vaccination coverage is high in every Latin American country, though there may be sectors with low coverage. This has been acknowledged and should be solved by governments taking a firm position $^{22,23}$.

\section{Conclusions and recommendations of SLIPE's Workshop (Polio/2014)}

\section{Schedule with IPV}

- According to SLIPE, it is acceptable, without detriment to the immune response, that the child vaccinated with combined vaccines may receive more IPV doses than the ones recommended in the primary schedule.

The optimal proposed schedule consists of four IPV doses (three doses in the primary schedule plus a booster dose), whether IPV is combined or not with other indicated vaccines in the immunization program of the country.

- During the OPV to IPV transition phase, it should be considered that:

a) It's possible to choose a schedule that combines the first 2 or 3 IPV doses followed by one or two OPV doses. 
b) For the 4-year-old dose, IPV or OPV can be administered. The latter can be administered in the routine schedule or through massive strategies during National Immunization Days.

- Children vaccinated with OPV, regardless of their prior schedule, should complete their immunization against poliomyelitis with IPV.

- IPV can be administered in its monocomponent presentation or as combined vaccines in any of the doses.

- Children who have been correctly and completely vaccinated with IPV don't need subsequent doses of polio vaccine for individual protection.

- Any schedule containing OPV, regardless of being tri-, bi- or monovalent, should be later discontinued to be replaced with a complete schedule with IPV, since, if the attenuated vaccine continues to be administered, the virus will keep circulating.

\section{Coverage}

- Countries should set optimal strategies in order to maintain and improve vaccination coverage.

- The number of low-coverage sectors in several countries, including displaced populations, should be reduced.

- A nominal immunization registry should be implemented.

\section{Epidemiological and Environmental Surveillance}

- Improving the Epidemiological Surveillance of Acute Flaccid Paralysis (AFP) according to WHO criteria.

- Setting up an environmental surveillance program to obtain data of the virus circulation.

\section{Strategies for introducing IPV in National Immunization Programs}

- Communicating properly with the population, considering the following aspects:

- Using language that is easy to understand in every social level.

- Facilitating communication with all the media (including the press).

- Optimizing the use of social networks as a tool to spread messages, especially among young people.

- Asking for the support of scientific societies in communications to establish higher credibility.

- Focusing communication on keeping population confident in immunization programs, including polio.

- Aligning the messages of the scientific societies and health ministries of the countries.

- Bringing the scientific societies closer to the decision makers of ministries and civil societies of the countries.
- As regards coming closer to the decision makers, scientific societies can participate in consulting committees or other kinds of groups of experts.

- Regarding civil societies, important scientific support can be given.

- SLIPE, as an international society, should continue to collaborate with the scientific societies of countries requesting support.

- Formally requesting PAHO's collaboration with SLIPE, in preparing for IPV introduction by means of: - more technical/academic support in the subject;

- coming closer to the scientific societies of the countries;

- including experts from SLIPE as part of the TAG.

- Ensuring that vaccine suppliers will offer optimal supply and prices for IPV introduction to guarantee the program sustainability.

- Setting up a schedule to incorporate IPV (and discontinue OPV), thus anticipating the new demands and informing in advance IPV requirements to suppliers.

- Urging suppliers to inform in good time any limitation that should arise in the vaccine supply.

- Negotiating prices that are feasible for IPV introduction, both as a combined vaccine and as a monocomponent.

- Training vaccination teams as an important source of information and program implementation.

- It is recommended to start training those with more experience, knowledge and leadership so that they can later help training the rest.

- Enhancing the distribution and storing logistics of vaccines, especially as regards maintaining the cold chain.

\section{Final Considerations}

We are very close to eradicating polio. It's not fair for children that this need is not understood, and that a vaccine, which can cause by itself the disease that is being combated, is still being used.

In addition to this scientific evidence, the countries that have not yet decided to switch to IPV should consider the implications of the prior item for equity and social justice reasons.

It's important to make it clear that the schedule proposed by SLIPE is a recommendation and that the countries will make the decisions according to their needs. Clearly, each country should have a budget assigned to the immunization programs, including the introduction of new vaccines and the sustainability of these programs. It should also be remembered that each Immunization Day 
with the attenuated oral vaccine costs millions of dollars and the known loss of oral vaccine is very big, which increases significantly the cost (in order to administer one dose, several doses are lost).

In this process, there are challenges and difficulties. The purpose of this meeting was to contribute to evaluate switching to IPV, sharing experiences and allowing the exchange of ideas between countries that have already changed their schedules and countries that haven't done it yet.

Now, everybody's collaboration is essential in this path towards the eradication of polio, caused both by the wild virus and the vaccine virus.

It's worth emphasizing WHO's recommendation that, by 2015 , every country should have included at least one dose of IPV in their vaccination programs. Besides, countries need to set up a schedule to interrupt the use of OPV and continue with IPV, either as a monocomponent or as a combined vaccine.

\section{Only by eradicating polio we can end inequity and promote social justice!}

Acknowledgements: To all the participants of the meeting who, as representatives of their entities, collaborated and contributed their experience and opinions to make it possible to prepare this declaration:

- SLIPE members, polio experts from Argentina, Brazil, Colombia, Costa Rica, the United States, Mexico and Dominican Republic;

- Members of the Expanded Program on Immunization from: Argentina, Brazil, Chile, El Salvador, Honduras, Mexico, Panama, Paraguay, Peru and Uruguay; representatives of American Academy of Pediatrics.

The sponsorship of Americas Health Foundation-AHF (Washington DC, United States).

To Ms. Herlys Gianelli, for collaborating during the meeting, gathering information and writing the conclusions and recommendations.

To Ms. Valeria Castro, for her contribution in the revision of the document.

\section{Abstract}

Even though we have already covered $99 \%$ of the path to eradicate poliomyelitis from the world, this disease is still causing paralysis in children. Its eradication means not only the end of wild poliovirus circulation, but vaccine-derived poliovirus circulation as well. Taking into account different factors such as: current epidemiological data, adverse events of the attenuated oral poliomyelitis vaccine (OPV), the availability of an injectable inactivated vaccine (IPV) without the potential of causing the severe adverse events of the oral vaccine (OPV), the efficacy and effectiveness of the IPV in several countries of the world where it has been used for several years, the rationale of changing the vaccination schedule in different Latin American countries; the Latin American Society of Pediatric Infectious Diseases (SLIPE) announces its recommendation of switching to IPV in Latin America, by this Declaration, with an Action Plan for 2014-2015 period as regards vaccination against polio policies in Latin America. 1. The optimal proposed schedule consists of four IPV doses (three doses in the primary schedule plus a booster dose), whether IPV is combined or not with other indicated vaccines in the immunization program of the country. During the OPV to IPV transition phase, an alternative schedule is acceptable; 2. Countries should set optimal strategies in order to maintain and improve vaccination coverage, and implement a nominal immunization registry; 3 . Improving the Epidemiological Surveillance of Acute Flaccid Paralysis (AFP) and setting up an environmental surveillance program; 4. Setting up strategies for introducing IPV in National Immunization Programs, such as communicating properly with the population, among others; 5 . Bringing scientific societies closer to decision makers; 6. Ensuring optimal supply and prices for IPV introduction; 7. Training vaccination teams; 8. Enhancing the distribution and storing logistics of vaccines. In addition to the scientific evidence, the countries that have not yet decided to switch to IPV should consider the implications of equity and social justice.

\section{References}

1.- WHO: Ten facts on Polio eradication. http:// www.who.int/features/factfiles/polio/en/. Available 26-04-14

2.- WHO-Poliomyelitis: intensification of the global eradication initiative-Report by the Secretariat. Sixty Seventh World Health Assembly-March 21, 2014.

3.- Global Polio eradication Initiative. Wild Polio Cases. http://www.polioeradication. org/Dataandmonitoring/Poliothisweek.aspx.
Consultado: 25-05-14

4.- World Health Organization-Poliomyelitis. http:// apps.who.int/gb/ebwha/pdf_files/WHA67/ A67_38-en.pdf. (Accessed April, 19, 2014).

5.- Global Polio eradication Initiative. Polio. Data and Monitoring. (http://www.polioeradication. org/Dataandmonitoring.aspx). (Accessed April, 19, 2014).

6.- World Health Organization. Polio eradication. Fact Sheets número 114. http://www.wh.int/ mediacentre/factsheets/fs114/en/. (Accessed April, 19, 2014).
7.- Pakistani City Is World's Biggest Reservoir of Polio Viruses. Medscape. Jan 17, 2014).

8.- $\quad$ Sutter R W, John T J, Jain H, Agarkhedkar S, Ramanan P V, et al. Immunogenicity of bivalent types 1 and 3 oral poliovirus vaccine: a randomized, double-blind, controlled trial. Lancet 2010 nov 13; 376 (9753): 1682-8. doi: 10.1016/S0140-6736(10)61230-5. Epub 2010 oct 25 .

9.- Paul Y. Polio eradication: experts have misled us. Medical Veritas 3 (2006) 781-5.

10.- Landaverde J M, Trumbo S P, Danovaro- 
Holliday M C, Cochi S E, Gandhi R, et al. Vaccine-associated paralytic poliomyelitis in the post elimination era in Latin America and the Caribbean, 1992-2011. J Infect Dis 2014 may; 209 (9): 1393-402. doi: 10.1093/infdis/jit602. Epub 2014 feb 11.

11.- Centers for Disease Control and Prevention. MMWR Morb Mortal Wkly Rep July 1, 2011; 60: 846-50. Update on VaccineDerived Polioviruses-Worldwide, April 2011-June 2012.

12.- Centers for Disease Control and Prevention. MMWR Morb Mortal Wkly Rep. Update on Vaccine-Derived Polioviruses-Worldwide, April 2011-June 2012. 2012; Sept 21, 61 (37); 741-6.

13.- Wringe A, Fine PE, Sutter RW, Kew OM. Estimating the extent of vaccine-derived poliovirus infection. PLOSOne. 2008; 3 (10): e3433. doi: 10.1371/journal.pone.0003433. Epub 2008 Oct 29.

14.- Global Polio Eradication Initiative. Acute Flaccid Paralysis (AFP) surveillance.
Consultado: http://www.polioeradication. org/Dataandmonitoring/Surveillance.aspx . (Accessed May, 21, 2014) .

15.- Global Polio eradication Initiative. Update on Vaccine-Derived Polioviruses-Worldwide, April 2011-June 2012WeeklySeptember 21, 2012/61(37); 741-6.

16.- World Health Organization. Polioeradication http://www.polioeradication.org/ Resourcelibrary/Strategyandwork/ EmergencyActionPlan.aspx. (Accessed April, 19, 2014).

17.- WHO-Sixty-Seventh World Health Assembly-Poliomyelitis: intensification of the global eradication initiative - 21 March 2014.

18.- WHO-SAGE - Global Polio eradication Initiative-Meeting November 2012. http://www. who.int/immunization/sage/meetings/2012/ november/Yellow_book_SAGE_Nov_2012_ final.pdf?ua=1. (Accessed April, 26, 2014).

19.- Source: Population Reference Bureau, 2011 http://www.prb.org/pdf11/2011 population-data- sheet_sp.pdf.

20.- Ministerio da Saude do Brasil. Vacinaçao. http:// www.brasil.gov.br/saude/2009/12/campanhasde-vacinacao-2. (Accessed May, 25, 2014).

21.- Ministerio de la Salud de Costa Rica. Vigilancia de Salud. http://www.ministeriodesalud. go.cr/index.php/vigilancia-de-la-salud/iniciovigilancia-normas-protocolos-guias-ms. Consultado: el 20 de mayo de 2014.

22.- Ministerio de la Salud de México. Esquemas de Vacunación. http://censia.salud.gob. $\mathrm{mx} /$ contenidos/vacunas/esquemavacunas. htmlAvailable 20-05-14

23.- WHO/PAHO-Immunization in the Americas2013-Summary. Comprehensive Family Immunization Family, Gender and Life Course. (Accessed April, 27, 2014).

24.- WHO PAHO-Health Situation in The Americas: Basic Health Indicators 2013. http://www. paho.org/hq/index.php?option=com_co ntent\&view $=$ article $\&$ id $=2470 \% 3$ Adatastatistics\&catid $=1900 \% 3$ Adata-statisticshome\&Itemid $=2003 \&$ lang $=p t$ 